\title{
Thermal OH (1667/65 MHz) Absorption and Nonthermal OH (1720 MHz) Emission Towards the W28 Supernova Remnant
}

\author{
F. Yusef-Zadeh \\ Department of Physics and Astronomy, Northwestern University, Evanston, IL. 60208 \\ (zadeh@northwestern.edu) \\ M. Wardle \\ Department of Physics, Macquarie University, NSW 2109, Australia (wardle@physics.mq.edu.au) \\ D.A. Roberts \\ Department of Physics and Astronomy, Northwestern University, Evanston, IL. 60208 \& Adler \\ Planetarium, 1300 S. Lake Shore Drive, Chicago, IL. 60605 (doug-roberts@northwestern.edu)
}

Received __; accepted _ 


\begin{abstract}
The W28 supernova remnant is an excellent prototype for observing shocked gas resulting from the interaction of supernova remnants (SNRs) and adjacent molecular clouds (MCs). We present two new signatures of shocked molecular gas in this remnant. One is the detection of main-line extended $\mathrm{OH}(1667 \mathrm{MHz})$ absorption with broad linewidths. The column density of $\mathrm{OH}$ estimated from the optical depth profiles is consistent with a theoretical model in which $\mathrm{OH}$ is formed behind a C-type shock front. The second is the detection of extended, weak $\mathrm{OH}(1720 \mathrm{MHz})$ line emission with narrow linewidth distributed throughout the shocked region of W28. These give observational support to the idea that compact maser sources delineate the brightest component of a much larger region of main line $\mathrm{OH}$ absorption and nonthermal $\mathrm{OH}$ $(1720 \mathrm{MHz})$ emission tracing the global structure of shocked molecular gas. Main line $\mathrm{OH}(1665 / 67)$ absorption and extended $\mathrm{OH}(1720 \mathrm{MHz})$ emission line studies can serve as powerful tools to detect SNR-MC interaction even when bright $\mathrm{OH}(1720 \mathrm{MHz})$ masers are absent.
\end{abstract}

Subject headings: ISM: Clouds - ISM: general - shock waves - supernova remnants-Xrays: ISM 


\section{Introduction}

$\mathrm{OH}(1720 \mathrm{MHz})$ masers unaccompanied by masing in the 1665 and $1667 \mathrm{MHz}$ main lines occur where supernova remnants (SNRs) interact with molecular clouds (MCs) (Frail, Goss \& Slysh 1994; Yusef-Zadeh et al. 1996; Green et al. 1997; Wardle and Yusef-Zadeh 2002). These so-called SNR masers are collisionally pumped within a shock wave driven into a dense molecular cloud. The $\mathrm{OH}$ molecule is believed to be produced by the indirect dissociation of water formed within a C-type shock (Lockett, Gauthier \& Elitzur 1999) by X-ray photons from the interior of the supernova remnant (Wardle 1999).

Despite several radio surveys to find SNR-MC interaction sites in the Galaxy, OH $(1720 \mathrm{MHz})$ masers have been detected toward only 19 SNRs, about 10\% of the total number of observed SNRs (Frail et al. 1997; Koralesky et al. 1998; Green et al. 1997; Yusef-Zadeh et al. 2002). This low detection rate is partly due to the fact that $\mathrm{OH}(1720 \mathrm{MHz})$ masers only arise under restrictive conditions in density and temperature (Lockett et al. 1999) and that velocity coherence and maximum pathlength for amplification are achieved at the limb of the remnant where the shock

propagates in a direction perpendicular to the line of sight. However, the $\mathrm{OH}$ abundance increases throughout the shocked region and is expected to be more extensive and less restrictive in its physical conditions (Wardle 1999) than the regions where bright maser spots occur.

The $\mathrm{OH}$ column densities required for the formation of $\mathrm{OH}(1720 \mathrm{MHz})$ masers $\left(z 10^{16} \mathrm{~cm}^{-2}\right)$ should be sufficient to create detectable thermal $\mathrm{OH}$ absorption in the main line transitions of $\mathrm{OH}$ $(1665 / 67 \mathrm{MHz})$ molecule if the background continuum is strong. In order to test this idea, we observed a nearby interacting SNR, W28 at the distance of $1.8 \mathrm{kpc}$ (Rho et al. 1994). W28 is a prototype SNR maser with numerous compact and bright maser spots distributed throughout the remnant (Claussen et al. 1997). OH absorption towards W28 has previously been detected with single-dish observations (Pastchenko \& Slysh 1974) and with the Australian Telescope Compact Array (Green et al. 2000). Here we report the VLA detection of broad OH (1667 MHz) absorption lines against the prominent W28 SNR continuum source. The column density of shocked OH gas estimated from optical depth profiles toward W28 is consistent with the theoretical prediction. In 
addition, we present extended, weak $\mathrm{OH}(1720 \mathrm{MHz})$ maser emission from W28. We then suggest that studies of thermal $\mathrm{OH}(1667 \mathrm{MHz})$ absorption and extended nonthermal $\mathrm{OH}(1720 \mathrm{MHz})$ emission can be used to search for additional SNR-MC interactions where compact $1720 \mathrm{MHz}$ masers are absent.

\section{Observations}

The Very Large Array (VLA) of the National Radio Astronomy Observatory ${ }^{1}$ was used on October 7, 2001 and July 18, 2000 to observe $\mathrm{OH}$ (1667 MHz) absorption and $\mathrm{OH}(1720 \mathrm{MHz})$ emission line studies, respectively. We used the compact D configuration to observe $\mathrm{OH}$ (1667 $\mathrm{MHz}$ ) line absorption toward W28 at center velocities of $10 \mathrm{kms}^{-1}$. Mode 4 of the correlator was used with 64 channels per IF; each IF pair is tuned to the different line transitions of OH, (1667 $\mathrm{MHz}$ and $1720 \mathrm{MHz}$ ). The channel separation was $6.104 \mathrm{kHz}$, which corresponds to $0.55 \mathrm{~km} / \mathrm{s}$. Standard calibrations of complex gains and bandpass were carried out using 1328+307, 1730-130 and 1851-235. Because of a strong negative bowl produced surrounding the continuum image of W28, a zero-spacing flux of 60 Jy was added to the continuum $u v$ data. The $u v$ data were then self-calibrated before the final line (l) and continuum (c) images were CLEANed. The rms noise in a given channel of W28 is $5.5 \mathrm{mJy}$ beam $^{-1}$. An optical depth $(\tau)$ image and corresponding error images were constructed for W28 by using $\tau=-\ln ((l+c) / c)$.

In the second VLA observation, we carried out an $\mathrm{OH}(1720 \mathrm{MHz})$ emission line study of W28. Unlike the previous high-resolution snapshot $\mathrm{OH}(1720 \mathrm{MHz})$ observations of W28 using the A-configuration of the VLA (Frail et al. 1994; Claussen et al 1997), we searched for extended, weak $\mathrm{OH}(1720 \mathrm{MHz})$ emission from W28 using the compact D configuration with a relatively uniform $u v$ coverage so as to be sensitive to extended but narrow line $\mathrm{OH}(1720 \mathrm{MHz})$ emission features. Mode $2 \mathrm{AC}$ of the correlator was chosen with 128 channels, a channel width of $1.06 \mathrm{~km} \mathrm{~s}^{-1}$

\footnotetext{
${ }^{1}$ The National Radio Astronomy Observatory is a facility of the National Science Foundation, operated under a cooperative agreement by Associated Universities, Inc.
} 
and a total bandwidth of $0.7812 \mathrm{MHz}$. This observation used similar phase and flux calibrators to that of absorption line observations. The rms noise for a given channel is $6.2 \mathrm{mJy} \mathrm{beam}^{-1}$. The rms noise for three velocity channels at $9.6,10.7$ and $11.8 \mathrm{kms}^{-1}$ where the most intense maser emission is observed were three times higher.

\section{Results}

\subsection{W28 (SNR G6.4-0.1)}

\subsubsection{Broad Thermal $\mathrm{OH}(1667 \mathrm{MHz})$ Absorption}

The right panel of Figure 1 shows a contour representation of $\mathrm{OH}(1667 \mathrm{MHz})$ line absorption toward W28 integrated between -3.2 and $23.7 \mathrm{kms}^{-1}$. The grayscale image shows the continuum image of W28 constructed from line-free channels. Much of the absorption is distributed prominently to the northeast of the remnant where background continuum is strongest. The absorbing gas velocities are very similar to $\mathrm{OH}(1720 \mathrm{MHz})$ maser velocities ranging between +4.8 and $+16 \mathrm{kms}^{-1}$ as reported by Claussen et al. (1997) and Frail et al. (1994). The panel to the left of the remnant in Figure 1 shows a typical absorption spectrum (position is indicated by the cross on the right panel) with a total linewidth of $\approx 30 \mathrm{kms}^{-1}$.

Figure 2 shows a grayscale continuum image at $20 \mathrm{~cm}$ (Dubner et al. 2000) with the distribution of compact $\mathrm{OH}(1720 \mathrm{MHz})$ maser spots represented as crosses (Claussen et al. 1997) superposed. The insets to the left and top of Figure 2 show the fitted optical depth profiles and their corresponding residuals. The optical depth velocity profiles coincide with clusters of $\mathrm{OH}$ $(1720 \mathrm{MHz})$ maser sources identified by Claussen et al. (1997). Gaussian fitted spectra of the top

and left $\tau$ profiles show center velocities between $\mathrm{V}_{L S R}=10.2$ and $8.1 \mathrm{kms}^{-1}$, which are similar to the velocities of of $\mathrm{OH}(1720 \mathrm{MHz})$ maser spots identified in the F, C and D regions of Claussen et al. (1997). The linewidths (FWHM) of 14 and $11 \mathrm{kms}^{-1}$ are much larger than typical linewidths of ambient molecular clouds, thus confirming the interaction picture of W28 with its surrounding 
molecular cloud (e.g. Wootten 1981). Table 1 gives the parameters of the fits to four $\tau$ spectra, all of which show broad line widths ranging between 7 and $10 \mathrm{kms}^{-1}$. However, the top-right spectrum of Figure 2 shows two narrow absorption lines with $\tau=0.23$ and 0.28 plus a broad absorption feature with $\tau=0.08$ at centroid velocity of $10 \mathrm{kms}^{-1}$. This spectrum is seen against a bright compact continuum source G6.6-0.1 $\alpha, \delta(\mathrm{J} 2000)=18^{\mathrm{h}} 00^{\mathrm{m}} 49.8^{\mathrm{s}},-23^{\circ} 20^{\prime} 24^{\prime \prime} .56$ showing a flux density of 0.76 Jy near the center of W28. This source is known to have a flat spectral index (Andrews et al. 1983; Dubner et al. 2000). The line profiles show FWHM linewidths of 1.5-1.8 $\mathrm{kms}^{-1}$ and are centered at 12 and $18 \mathrm{kms}^{-1}$. The narrow line widths of absorption features suggest that they are not associated with the SNR-MC interaction. The high velocity component is close to the high velocity component of HI absorption measurements (Radhakrishnan et al. 1972). Claussen et al. (1997) suggest that the systemic velocity of the molecular cloud associated with W28 is at $17.6 \mathrm{kms}^{-1}$ and that the low-velocity $7 \mathrm{kms}^{-1}$ component is being accelerated by the SNR shock along the line of sight by about $-10 \mathrm{kms}^{-1}$. This suggests that the narrow line spectra observed in Figure 2 are associated with the MC in the vicinity of W28.

A bright, compact $\mathrm{OH}(1667 \mathrm{MHz})$ maser source with two velocity components is detected in the vicinity of $\mathrm{W} 28$. This source lies at $\alpha, \delta(\mathrm{J} 2000)=18^{\mathrm{h}} 01^{\mathrm{m}} 57.78^{\mathrm{s}},-23^{\circ} 12^{\prime} 31^{\prime \prime} .43$. The spectrum shows two narrow velocity components at $17.1 \mathrm{kms}^{-1}$ and $23.5 \mathrm{~km} \mathrm{~s}^{-1}$ with a flux density of 0.39 and $0.05 \mathrm{Jy}$, respectively, resembling the spectrum of $\mathrm{OH} / \mathrm{IR}$ stars.

\subsubsection{Extended Maser Emission}

Figure 3 shows contours of the $\mathrm{OH}(1720 \mathrm{MHz})$ line emission integrated between 5.3 and 21.3 $\mathrm{km} / \mathrm{s}$ (with the exception of three channels at 9.6, 10.6 and $11.7 \mathrm{kms}^{-1}$, which were contaminated by saturation from strong maser emission) superposed on a grayscale continuum image of W28. The crosses indicate the positions of 41 compact maser sources listed by Claussen et al. (1997). We note extended and compact $\mathrm{OH}(1720 \mathrm{MHz})$ emission from W28. The top two insets show the velocity profiles of two isolated sources to the north of the shell. These sources, as well as extended maser emission adjacent to compact maser sources, have not been detected in earlier 
high resolution observations which over-resolved these extended features. The third OH (1720 $\mathrm{MHz}$ ) velocity profile in the bottom left panel of Figure 3 coincides with the position of the optical depth profile presented in the top left panel of Figure 2. It is clear that where $\mathrm{OH}(1667 \mathrm{MHz})$ absorption profile is broad, $\mathrm{OH}(1720 \mathrm{MHz})$ line emission is narrow.

The extended $\mathrm{OH}(1720 \mathrm{MHz})$ emission features have typical linewidths of about 1-3 $\mathrm{kms}^{-1}$. The flux density of extended features are typically about 100-300 mJy/beam corresponding to a brightness temperature $\mathrm{T}_{b} \sim 20-60 \mathrm{~K}$. The kinematics of extended $\mathrm{OH}(1720 \mathrm{MHz})$ emission is very similar to that of compact masers, suggesting that they co-exist with each other. The extended features with their nonthermal characteristics trace shocked CO (3-2) molecular gas toward W28 (Arikawa et al. 1999) in spite of their low brightness temperatures.

\section{Discussion}

The column density of $\mathrm{OH}$ toward W28 may be estimated from the observed optical depth velocity profiles using

$$
N_{\mathrm{OH}}=2.2785 \times 10^{14} T_{\mathrm{ex}} \int \tau_{v} d v \quad \mathrm{~cm}^{-2}
$$

for the $1667 \mathrm{MHz}$ line once a value for the excitation temperature, $T_{\mathrm{ex}}$, has been chosen (Crutcher 1977). Because the energy spacing between the upper and lower levels is $\approx 0.08 \mathrm{~K}, T_{\mathrm{ex}}$ is sensitive to small departures of the level populations from LTE. In order to estimate $T_{\mathrm{ex}}$, we modelled $\mathrm{OH}$

level populations in a uniform slab of gas with a FWHM linewidth of $10 \mathrm{kms}^{-1} . T_{\text {ex }}$ is determined largely by the kinetic temperature $T_{k}$ and is insensitive to density and column density for the parameters that are relevant here. The excitation temperature is close to the kinetic temperature $T_{k}$ for $T_{k} \lesssim 20 \mathrm{~K}$ and then decreases with increasing kinetic temperature as collisions begin to populate the higher rotational states: $T_{\mathrm{ex}}=18,8$, and $3 \mathrm{~K}$ at $T_{k}=30,50 \mathrm{~K}$ and $75 \mathrm{~K}$ respectively. Therefore we adopt $T_{e x}=10 \mathrm{~K}$ as a nominal value with the assumption that $T_{e x}$ may differ by a factor of two in either direction. Our measurements indicate that $\int \tau_{v} d v \approx 10 \mathrm{kms}^{-1}$, therefore $N_{\mathrm{OH}} \approx 2 \times 10^{16} \mathrm{~cm}^{-2}$. This $\mathrm{OH}$ column density is consistent with that required to produce 1720 
MHz maser emission (Lockett, Gauthier \& Elitzur 1999) and expected from X-ray dissociation of water produced in a C-type shock wave driven into the molecular cloud by the supernova remnant (Wardle 1999).

Extended $1720 \mathrm{MHz}$ emission can be produced by weak amplification in the $\mathrm{OH}$ behind a face-on shock front as opposed to the edge-on geometry required by the bright maser spots. The brightness temperature of the $1720 \mathrm{MHz}$ emission, $\sim 30 \mathrm{~K}$ at line center, is roughly equal to that of the background continuum. The implied amplification factor of 2 requires an $\mathrm{OH}$ column $N_{\mathrm{OH}} \approx 10^{15} \mathrm{~cm}^{-2}$ with $T_{k} \gtrsim 50 \mathrm{~K}$ (Lockett et al 1999 ), and line FWHM $\lesssim 0.5 \mathrm{kms}^{-1}$, conditions that are achieved in the cooling tail of a C-type shock wave (Wardle 1999). It should be possible to detect this weakly-masing component in absorption at $1667 \mathrm{MHz}$, as the optical depth is $\approx 2$ at line center. $\mathrm{H}_{2} \mathrm{CO}$ absorption study of W28 also indicates that the interacting cloud has a low kinetic temperature and that the properties of the interacting cloud are consistent with the picture of cold post-shocked gas (Slysh et al. 1980).

The observations presented here trace extended regions of shocked molecular gas in which the abundance of $\mathrm{OH}$ has been enhanced. Our results suggest two new ways to search for SNR-MC interaction sites where $\mathrm{OH}(1720 \mathrm{MHz})$ masers are absent. One is to search for extended $\mathrm{OH}(1720$ $\mathrm{MHz}$ ) maser emission produced by shock waves. Unlike bright, compact masers this emission does not require the shock to propagate almost perpendicular to the line of sight, and should therefore be more generic. This emission has been detected in the five SNRs with compact masers that have been searched to date (Yusef-Zadeh et al. 1995; 1999; and this paper). SNR-molecular cloud interaction sites can also be identified by searching for broad $\mathrm{OH}$ absorption lines at $1665 / 67 \mathrm{MHz}$ delineating the region where shocked molecular gas overlies the radio continuum emission from the SNR. A strong empirical association between X-ray emitting centrally-filled mixed-morphology remnants and maser-emitting shell-type SNRs has recently been demonstrated by Yusef-Zadeh et al. (2002). Remnants that show centrally filled thermal X-ray emission are strong candidates for such studies.

Acknowledgments: We thank G. Dubner for providing us with the continuum image of W28. 


\section{REFERENCES}

Arikawa, Y., Tatematsu, K., Sekimoto, Y. \& Takahashi, T. 1999, PASJ, 51, L7

Andrews, M.D., Basart, J.P., Lamb, R.C. \& Becker, R.H. 1983, ApJ, 266, 684

Claussen, M.J., Frail, D.A., Goss, W.M., \& Gaume, R.A. 1997, ApJ, 489, 143

Crutcher, R.M. 1977, ApJ, 216, 308

DeNoyer, L. K. 1979, ApJ, 228, L41

Dubner, G. M., Velzquez, P. F., Goss, W. M. \& Holdaway, M. A 2000, AJ, 120, 1933

Frail, D. A., Goss, W. M., Reynoso, E. M., Giacani, E.B., Green, A. J. \& Otrupcek, R. 1996, AJ, 111,1651

Frail, D.A., Goss, M.W. \& Slysh, V.I. 1994, ApJ, 424, L111

Giacani, E.B., Dubner, G.M., Kassim, N.E., Frail, D.E., Goss, W.M. et al. 1997, AJ, 113, 1379

Green A.J., Frail, D.A., Goss, W.M. \& Otrupcek, R. 1997, AJ, 114, 2058

Green, A. J., Wardle, M. \& Lazendic, J. 2000, 24th meeting of the IAU held in Manchester, England.

Koralesky, B., Frail, D.A., Goss, W.M., Claussen, M.J. \& Green, A.J. 1998, AJ, 116, 1323

Lockett, P., Gauthier, E. \& Elitzur, M. 1998, ApJ, 511, 235

Pastchenko, M.I. \& Slysh, V.I. 1974, A\&A 35, 153

Radhakrishnan, V., Goss, W.M., Murray, J.D., \& Brooks, J.W. 1972, ApJS, 24, 49

Rho, J. Petre, R. Schlegel, E.M. \& Hester, J.J. 1994, ApJ, 430, 757

Slysh, V.I., Wilson, T.L., Pauls, T. \& Henkel, C. 1980, in IAU Symp. 87, 'Interstellar Molecules", eds: B.H. Andrew pp473-478

Wardle, M. 1999, ApJ, 525, 101.

Wardle, M. \& Yusef-Zadeh, F. 2002, Science, 296, 2350 
Wootten, A. 1981, ApJ, 245, 105

Yusef-Zadeh, F., Uchida, K.I., \& Roberts, D.A. 1995, Science 270, 1801

Yusef-Zadeh, F., Roberts, D.A., Goss, W.M., Frail, D.A. \& Green, A. 1996, ApJ, 466, L25

Yusef-Zadeh, F., Roberts, D.A., Goss, W.M., Frail, D.A. \& Green, A. 1999, ApJ, 527, 172

Yusef-Zadeh, F., Wardle, M., Rho, J. \& Sakano, M. 2002, ApJ, submitted. 
Fig. 1. - [right panel] Contours of $\mathrm{OH}(1667 \mathrm{MHz})$ absorption toward W28 integrated between -2.1 and $23.7 \mathrm{kms}^{-1}$ at $-4.0,-2.0,-1.0,-0.5 \mathrm{mJy}_{\mathrm{beam}}{ }^{-1} \mathrm{kms}^{-1}$ are superposed on a grayscale continuum image with a spatial resolution of $68^{\prime \prime} \times 30^{\prime \prime}, \mathrm{PA}=40^{\circ}$. The range is grayscale brightness is between 0 and $270 \mathrm{mJy}$. [left panel] A typical spectrum showing an $\mathrm{OH}(1667 \mathrm{MHz})$ absorption line toward the cross at $\alpha, \delta(\mathrm{J} 2000)=18^{\mathrm{h}} 01^{\mathrm{m}} 41.43^{\mathrm{s}},-23^{\circ} 25^{\prime} 11^{\prime \prime} .3$.

Fig. 2.- A grayscale continuum image at $20 \mathrm{~cm}$ with a spatial resolution of $88^{\prime \prime} \times 48^{\prime \prime}$ (Dubner et al. 2000) with the distribution of compact $\mathrm{OH}(1720 \mathrm{MHz})$ maser spots represented as crosses (Claussen et al. 1997) superposed. The insets show the derived optical depths (including error bars), Gaussian fits, and corresponding residuals.

Fig. 3.- A grayscale continuum image of W28 at 20cm (Dubner et al. 2000) with superposed contours of $\mathrm{OH}(1720 \mathrm{MHz})$ maser emission set at $-3,3,5,7,9,11,13,15,20,25,30,40,50$, $60,70,80,100$ mJy beam ${ }^{-1}$ with a spatial resolution of $86.6^{\prime \prime} \times 34.6^{\prime \prime}, \mathrm{PA}=14^{\circ}$ (shown as the white ellipse in the bottom right hand corner). The crosses represent the position of compact OH (1720 MHz) masers identified by Claussen et al. (1997). The respective positions of the three spectra on the top, bottom left, and top left are: $\alpha, \delta(\mathrm{J} 2000)=18^{\mathrm{h}} 01^{\mathrm{m}} 43.6^{\mathrm{s}},-23^{\circ} 24^{\prime} 19^{\prime \prime} .8$, $18^{\mathrm{h}} 01^{\mathrm{m}} 43.33^{\mathrm{s}},-23^{\circ} 12^{\prime} 59^{\prime \prime} .2$ and $18^{\mathrm{h}} 01^{\mathrm{m}} 32.4^{\mathrm{s}},-23^{\circ} 11^{\prime} 10^{\prime \prime} .01$. 
Table 1. OH (1667 MHz) Optical Depth towards W28

\begin{tabular}{|c|c|c|c|c|}
\hline $\begin{array}{c}\text { Position } \\
(\mathrm{RA}, \text { Dec }(\mathrm{J} 2000))\end{array}$ & $\begin{array}{c}\text { Amplitude } \\
\text { (optical depth) }\end{array}$ & $\begin{array}{l}\text { Center Velocity } \\
\qquad\left(\mathrm{km} \mathrm{s}^{-1}\right)\end{array}$ & $\begin{array}{l}\text { FWHM Line Width } \\
\qquad\left(\mathrm{km} \mathrm{s}^{-1}\right)\end{array}$ & $\begin{array}{l}\text { Corresponding } \mathrm{OH} \\
(1720 \mathrm{MHz}) \text { masers }\end{array}$ \\
\hline \multirow[t]{3}{*}{$18^{\mathrm{h}} 00^{\mathrm{m}} 49.924,-23^{\circ} 20^{\prime} 26^{\prime \prime} 66$} & $0.229 \pm 0.017$ & $12.471 \pm 0.130$ & $4.439 \pm 0.387$ & A \\
\hline & $0.276 \pm 0.018$ & $18.870 \pm 0.093$ & $3.328 \pm 0.253$ & \\
\hline & $0.085 \pm 0.011$ & $9.983 \pm 1.156$ & $23.980 \pm 2.687$ & \\
\hline $18^{\mathrm{h}} 01^{\mathrm{m}} 50.002,-23^{\circ} 18^{\prime} 46^{\prime \prime} .58$ & $0.726 \pm 0.027$ & $10.269 \pm 0.183$ & $14.163 \pm 0.765$ & $\mathrm{~F}$ \\
\hline $18^{\mathrm{h}} 01^{\mathrm{m}} 44^{\mathrm{s}} .041,-23^{\circ} 24^{\prime} 23^{\prime \prime} 05$ & $1.148 \pm 0.075$ & $6.845 \pm 0.195$ & $8.928 \pm 0.489$ & $\mathrm{C}, \mathrm{D}$ \\
\hline $18^{\mathrm{h}} 01^{\mathrm{m}} 41^{\mathrm{s}} .444,-23^{\circ} 25^{\prime} 05^{\prime \prime} 26$ & $0.668 \pm 0.026$ & $8.533 \pm 0.170$ & $11.508 \pm 0.532$ & $\mathrm{C}, \mathrm{D}$ \\
\hline
\end{tabular}


This figure "fig1.gif" is available in "gif" format from: http://arxiv.org/ps/astro-ph/0209485v1 
This figure "fig2.gif" is available in "gif" format from: http://arxiv.org/ps/astro-ph/0209485v1 
This figure "fig3.gif" is available in "gif" format from: http://arxiv.org/ps/astro-ph/0209485v1 